\title{
An overview of STEAMER: An advanced computer-assisted instruction system for propulsion engineering
}

\author{
MICHAEL WILLIAMS \\ Xerox Palo Alto Research Center, 3333 Coyote Hill Road, Palo Alto, California 94304 \\ JAMES HOLLAN \\ Navy Personnel Research and Development Center, San Diego, California 92152 \\ and \\ ALBERT STEVENS \\ Bolt Beranek and Newman, Inc., 10 Moulton Street, Cambridge, Massachusetts 02238
}

\begin{abstract}
We are building a computer-based instructional system for teaching the operation of complex physical mechanisms. The system is based intimately on the use of simulation. Our goal in this paper is to describe the system we are attempting to construct, discuss its origins, and identify what we perceive to be its implications for psychological research into instruction.
\end{abstract}

Propulsion engineering is a major training problem for the U.S. Navy. Indeed, it is commonly judged by officers to be the single greatest training problem. Much of this is due to the complexity of the system. Not only are the propulsion plants physically large (approximately one third of the space on a ship), but they also comprise thousands of complex components (valves, pumps, tanks, etc.). The plants are operated by 10 to 20 watch standers who have prescribed sets of duties and work as a team. The team members range from the Engineering Officer of the Watch, through enlisted supervisors responsible for operations in various compartments, down to junior enlisted personnel who turn vaives, operate panels, keep logs, and so on. The primary function of all these operators is to control the plant, monitor its operation, and recover from any casualty conditions that might arise.

The operators of these plants must be trained to comprehend and carry out hundreds of procedures. A typical collection of operating procedures (in the form of annotated checklists) runs to two (or more) 4-in. thick volumes. Mistakes can lead to expensive damage and even deaths. Operators function in the midst of a vast control process that they all manipulate to some extent.

We are using a set of new technologies derived for work in artificial intelligence (AI) and cognitive science. These technologies make it possible to construct "intelligent" computer programs that can serve as tutors. Our goal is to build an intelligent computer-aided instruction (ICAI) training system to enhance Navy training in propulsion engineering. Primarily, the focus is on conceptual training. We want to train students in the primary principles of propulsion engineering. This is motivated by our belief that it is impossible to memorize all of the operating procedures. Furthermore, in many casualty situations, operators must literally invent the appropriate procedure to correct the casualty condition or to troubleshoot the steam plant. The system is referred to as STEAMER.

\section{STEAMER: THE BASIC IDEA}

Currently, there exists a full-scale mock-up simulator of a 1,200-psi steam plant. This system is located at the U.S. Navy Surface Warfare Officer School in Newport, Rhode Island. The essential idea in STEAMER is to take the math model that underlies this simulator, modify it, and use it as the basis for our development. We plan to put the modified model into a tabletop computer, provide a graphics interface to permit students access to inspect and manipulate the steam plant, and build an intelligent tutor to supervise the student's interaction and provide explanations. STEAMER is a 5-year project consisting of four major phases, each of which is designed to yield a useful product for use in Navy training.

Phase 1 of the project is the construction of a classroom demonstration system. This will be on a large computer of some kind. The system will consist of the math model with a graphics display. The display will be connected to a large screen projector and can be used by an instructor in a classroom setting to demonstrate operation of the propulsion plant. We envision this system as providing an experimental environment in which to collect data on the nature of explanation and the use of graphics to interface to the math model.

Phase 2 consists of taking the Phase 1 system and building in a limited tutor capability. We plan to use largely traditional computer-aided instruction (CAI) techniques. This system will serve as an in situ develop- 
ment environment, in which we intend to evolve an advanced ICAI tutorial system. The system will see limited classroom use with individual students. We expect that instructors will be a necessary backup to the system.

The Phase 3 system will consist of the Phase 2 system incorporated into a desk-top computer. The use of small machines will permit wider distribution of the training system.

Phase 4 is the development of the ICAI tutor. This phase is the most difficult and will run throughout the three other phases. The tutor will provide students with explanations of the performance of the system, identify and attempt to correct student misconceptions, and generally guide the student in his interaction with the system. The range to which we can distribute STEAMER is critically dependent on the degree of success of the intelligent tutor. It is this tutor that holds hope for sending the system to remote sites without instructor support (e.g., shipboard).

The in situ development scheme is, we believe, critical to keeping a project of this size on target to insure that it satisfies a legitimate training need. It is all too easy for scientists and programmers to spend excessive amounts of time solving problems that have lit tle relevance to real-world training concerns. It is our hope that the intimate contact with the clients will keep us on track.

In order to give you a more concrete notion of the STEAMER system, let us show you what a student interaction might look like. We have constructed a simplified simulation to demonstrate some of our notions about how the student should be given access to the simulation and to explore the nature of a graphics interface. This simulation is of a nuclear power plant that was chosen for historical reasons unrelated to our present effort.

While the diagrams that the student sees are in color, we have converted some to black-and-white line drawings to fit within the publication limitations of this paper. Where arrows are drawn to indicate flow, students see simulated fluid flows. System components are color coded to indicate state (e.g., open-shut, on-off, etc.).

The basic objective of a steam plant is to convert heat into electricity and to turn the ship's propeller. Heat is used to boil water in the steam generator. The steam is sent through the main steam header through a set of valves to the turbine. The turbine uses the steam to rotate a shaft that is used to generate electricity and to turn the ship's screw. The steam is then cooled in the main condenser and converted back into water. Sea water is used as the coolant. The water is pumped through a succession of pumps back to the steam generator to be boiled again.

The student can manipulate the steam plant by opening or shutting valves, or turning components on or off. He does so by touching the component on the screen and touching a command in a simple menu. Thus,

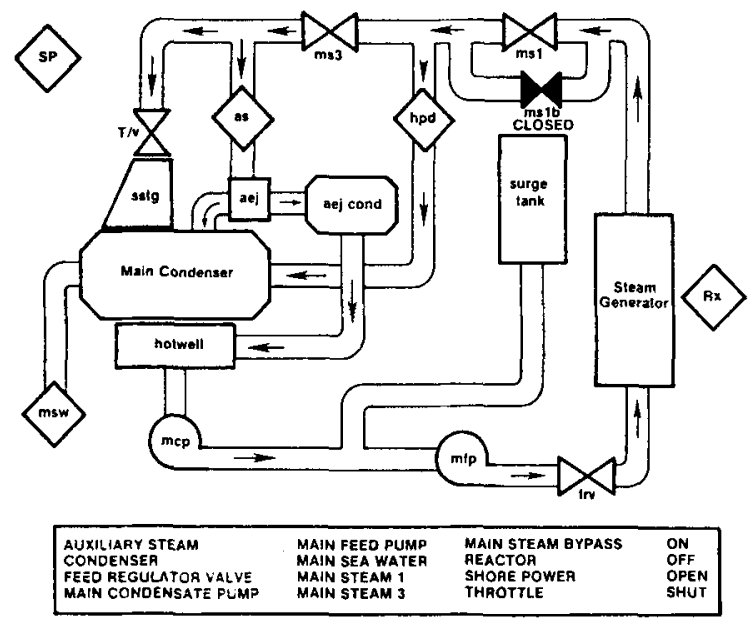

Figure 1.

in Figure 1 the student would open the main steam stop (MS-1) by touching MS-1 and the menu command "open." This permits the heating of the main steam header. One of the advantages of our simulation is that while it would normally take $15 \mathrm{~min}$ to heat the header, we can simply tell the student of the normal time delay and progress to the next part of the problem.

A change in state of a component is indicated by a color change. Thus when MS-1 is opened, the valve changes from red to green. Fluid flows are shown dynamically and fluid levels are shown in each tank. Thus the dead head of steam can be shown by the pipe's being filled with white. Steam flow is shown by the percolation of shades of white and gray through the pipe.

The interface to this simulation is extremely easy to use. The combined use of color and animation allows the student to easily see and understand the state of the simulated plant at any instant. Students with no background in computers or steam plants learn to use the simulation within a few minutes. It makes possible flexible interaction between the student and the tutor. If the student makes a mistake, the tutor can either prohibit the action and tell the student why or let the student see the natural consequences of the error.

We noted that steam plants are complex systems with thousands of components. The schematic available to the student in this example shows only 20 or so components. Each of the components in the diagram really represents an entire subsystem. The student has access to the subsystem parts by pointing to the subsystem and giving the command "expand." The system diagram is then replaced by a diagram of the subsystem selected. For example, if the student were to touch the component labeled AS (auxiliary steam), the auxiliary steam system diagram (see Figure 2) would be displayed. The student could then manipulate and observe the components of the auxiliary steam system.

The auxiliary steam system also consists of subsystems and components, some of which can in turn be expanded. Thus the student could look inside the auxiliary steam reducing valve (AS-1; Figure 3) and 


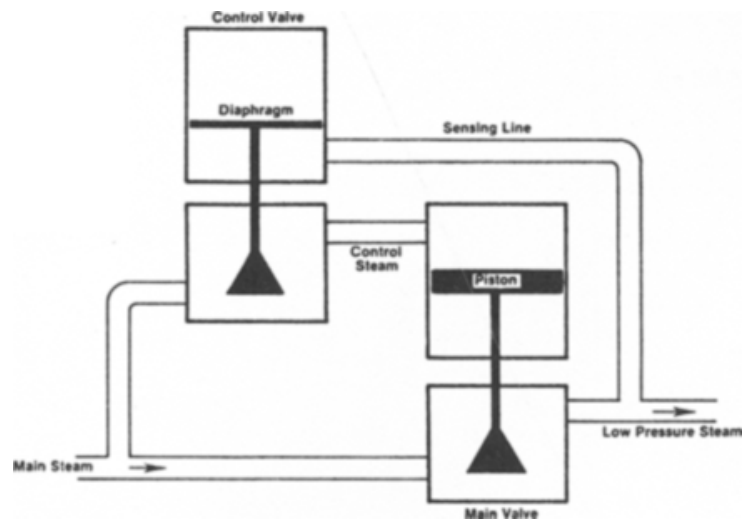

Figure 2.

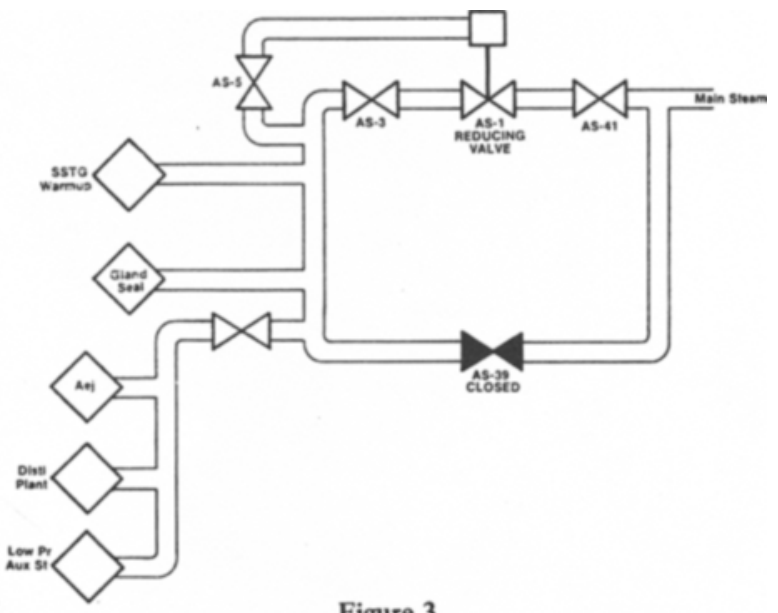

Figure 3.

observe the interaction of a pilot valve controlled by the feedback of the downstream pressure that actuates the main reducing valve.

The three primary things to notice about the interface are as follows. (1) The ease of use: it takes the student virtually no time to learn how to manipulate the plant. (2) The flexibility for the tutor: the tutor has the choice of advising the student at the time of the action or of letting the student make a mistake and see the consequences. (3) The use of what we call embedded simulation: not only can we give the student access to all of the math model, but we can also build microsimulations of individual components for the student to use to learn about the internal operation of those components.

We have built some sample embedded simulations to explore what is possible within the STEAMER framework. One possible notion is to build simulations that permit students to walk through the causal chain of operation of various components. For example, we have built a system that permits the student to walk through the feedback loop of the auxiliary steam reducing valve (Forbus \& Stevens, Note 1). This program actually generates a qualitative "explanation" from a topology of constraints in an underlying simulation. One instant out of a student interaction is shown in Figure 4. We cannot readily demonstrate in such a figure all of the graphics and animation tricks used to enhance the explanation for the student. Another use of embedded simulation is a "visible" venturi, which allows students to watch the flow in a venturi and to probe the velocity and pressure of a fluid at any point in the flow.

There is a set of additional capabilities we envision for STEAMER beyond those discussed above. In addition to the schematic diagram interface, the student has a second graphics interface. On this second terminal, the student has a dynamically controlled instrument display. Individual pressure gauges, tank level indicators, and so on, are presented in this space, as opposed to just the schematics. The second terminal also provides access to on-line documentation of those volumes of operating and casualty control procedures mentioned earlier.

Because the student is dealing with a simulation on which he is the only student and because a detailed trace of the interaction can readily be maintained by the computer, a replay facility is available in STEAMER. The replay can permit the student to return to a critical point to modify a decision and to follow the new consequences. Also, the tutor can replay the scenario and insert advice and critiques that would have been disruptive during the initial interaction.

A fourth capability we envision for STEAMER that is not present in the mock-up is what we call the super sensor system. The math model maintains numerous parameters for which there exists no instrumentation (e.g., steam flow from the main turbine, enthalpy of the steam in the main condenser). However, there is no reason the computer tutor cannot permit the student to observe these on an imaginary instrument. In addition, we want the student to be able to direct the tutor to maintain a graphical plot of these or any other system parameter during transients or to plot them against each other.

\section{UNDERLYING IDEAS}

There are three underlying ideas running throughout the STEAMER project that we believe give it the potential to change training in the Navy. Each seems independent from the others.

\section{Qualitative Reasoning}

We can all readily imagine taking a portable and inspectable simulation like this and just handing it to a student to explore the system. It is all too clear that there are a multitude of important training advantages possible with such a simulation, constraints inherent in an actual plant can be removed, time can be speeded up and slowed down, event sequences can be replayed, causality procedures too dangerous to attempt on a real plant can be readily practiced. But is that good enough? As cognitive psychologists our interest is how one might use such a capability with maximum instructional effectiveness. Ideally, in addition to the simula- 


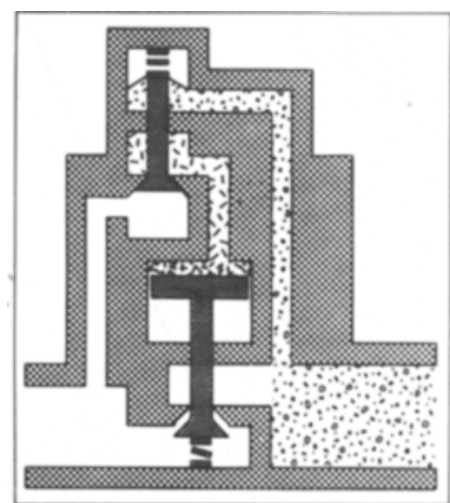

2. AND the PRESSURE IN CHAMBER 4 FALLS.

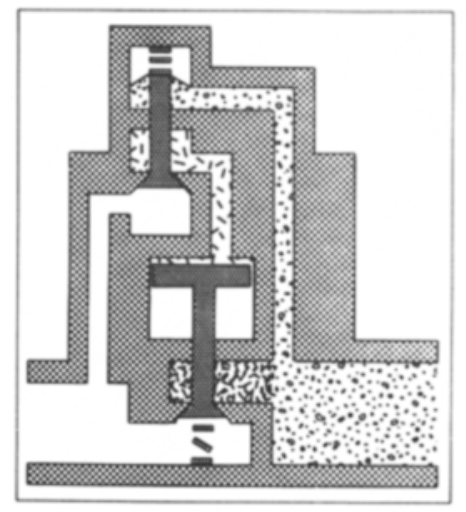

11. THE PAESSURE IN THE MAIN VALVES OUTPUT
SioE FALLS.

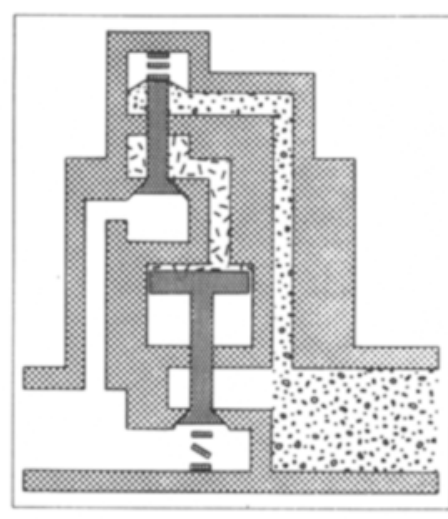

10. THE PISTON MOVES UP AND CLOSES THE MAIN VALVE.

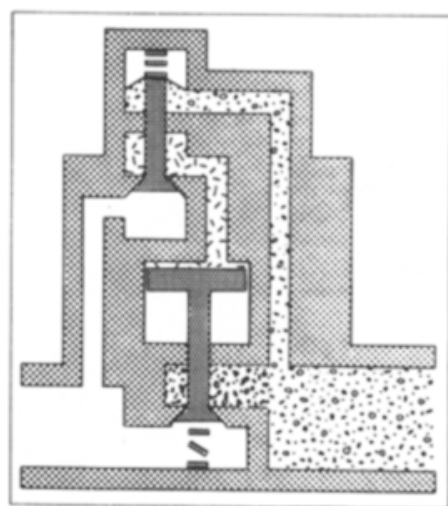

12. ALSO WHEN THE PRESSUAE IN CHAMBER O AISES THE PAESSURE IN THE MAIN VALVE'S OUTPUT SIOE RISES NOTICE THAT WHEN THE PRESSUAE IN THE OUTPUT VALT RISES IT MEANS THE PAESSUAE IN THE MAIN THE SYSTEM TO ACT SO THAT THE PRESSURE IN CAUSES THE MAIN VALVES OUTPUT SIDE FALLS. THIS ME ANS IT EXHIBITS NEGATIVE FEEDBACK.

Figure 4.

tion, one would like to provide the student with a private tutor to answer questions, provide hints, and give the explanations that are required to develop a deep understanding of the system being simulated. We think that an automated "intelligent" tutor might well be able to provide many of the same sort of hints and explanation. The integration of such a tutor with a simulation is central to the STEAMER project. We think that this will lead to an effective instructional system. It is our hope that the tutor will be able to explain the system to the student, to analyze the student's misunderstandings, and to guide the student in learning the conceptual principles of steam plant operations.

As we have already explained, a naval steam plant is an exceedingly complex system. We believe that it is difficult (perhaps impossible) to learn any steam plant's many operating procedures without some substantial amount of comparatively deep conceptual understanding of the devices and mechanisms that go into the design of the plant. There are just too many things to learn by rote. It is our view that the fundamental goal of a STEAMER system is to communicate this conceptual understanding.

The primary idea that gives us the confidence to attempt the development of such an automated tutor is the notion of qualitative reasoning. We have begun by looking at how people talk about and reason about steam plants. First, the kind of thing operators seem to have in their heads is more like a mental simulation than a list of facts or collection of procedures. Yet it is not very much like the quantitative. simulation that our math model is. Rather, people seem to use a qualitative "simulation." For example, when an operator talks 
about a changing water level in a tank, he makes comments like, "If the level is decreasing, then the water flow in has decreased or the flow out has increased."

This example illustrates one of the important components of qualitative reasoning: a device model. For a tank with an input and an output, the device model can tell us that if the level begins to decrease, then either the flow into the tank has decreased or the flow out has increased. Further, this calculus gives us very human ambiguities. If flow into and flow out are increasing, then one cannot tell which way the level is going. But one does know where to look: which increased more, flow out or flow in?

These notions of device models and qualitative reasoning have been developed over the last several years by deKleer $(1979 a, 1979 b)$ in the domain of electronics troubleshooting and reasoning about electronic circuits.

It turns out that the device model we have given here is not all there is. Many device models are actually derivable from embedded device models. Also, in the case of more complex device models, operators probably do learn some troubleshooting cases by rote. For example, for a heat exchanger, an operator will learn after some practice that a very likely source of the problem of insufficient cooling of the hot leg fluid is a restricted cold leg flow. This knowledge is, in some sense, compiled in and no longer dependent on any reasoning with the device model.

We do not wish to oversimplify the issue. Device models are not all there is to conceptual understanding of complex systems. Indeed, the explanations that can be generated with such models are often deceptively simple, in some cases wrong, and often ambiguous. We are particularly concerned that a student can listen to and comprehend a cogent explanation, leaving him the sense that he "understands" what is happening. However, when the student later tries to regenerate the explanation, he discovers he cannot do it, he has been tricked. Ambiguous decision points have been glossed over. He may even recall which way the explanation goes, but not really know why it goes that way.

On the other hand, there are a variety of reasons why we feel that these ideas hold particular promise. For one thing, the device models feel particularly good. That is, it seems that qualitative reasoning is precisely what operators use when thinking about steam propulsion plants. We get our information here from two sources. The personal testimonials of one of the authors (Mike Williams spent 5 years training on and operating nuclear-powered submarines with steam plants) and the responses of steam plant operators when we introduce the notion to them. These operators, when they have qualitative reasoning explained to them, characteristically respond with a positive "Yes, that's exactly it. That's the way I think about the plant."

Another reason that this notion of qualitative reasoning seems so attractive is that it provides a means of generating the complex procedures from a relatively compact set of models. It seems unlikely that anyone could memorize the hundreds of operating procedures needed to successfully operate the plant. Yet people seem to come close. Worse yet, the casualty conditions in which procedures must be invented on the fly means that operators need some sort of deeper understanding of the system. It seems that a simple rule-based system would be overloaded. There are more permissible states to a steam plant than there are protons in the universe.

One final reason that qualitative reasoning seems so attractive is that it lends itself to a type of embeddedness that seems so friendly to reasoning about steam plants. Thus, a device model is embedded with the heat exchanger device mode. And this underlying device model is made up of fragments that can be formed into the device models for an array of other components (Forbus \& Stevens, Note 1).

\section{The Graphics Interface}

One research issue that has drawn our attention is the use of graphics as an interface to the simulation. It seems to be incredibly easy to use. It takes just a few moments to instruct a computer-naive student in how the simulation uses the interface to operate the plant. We want to know why that is so. One could use a simple bandwidth argument, but we believe that is little more than restating the initial observation. We suspect it may have something to do with the explicit presentation of constraints on each of the components and the close relation between a graphical object and an object in the real world. In any case, one thing we want to do is take a shot at a theory of this kind of interface and understand the compelling role it can play in learning.

The graphics offer a unique kind of inspectability to the STEAMER system. Students can look "inside" a control valve or boiler to observe directly the interactions of fluids and mechanical components. Transient parameters can be plotted over time. The causal flow of events can be explicitly identified and displayed. Overviews beyond the scale available in traditional simulators is available. Mind you, each of these innovations requires substantial elaboration over the math model presently available. But each is possible with the high-level software development tools now available from recent work in $\mathrm{Al}$.

\section{Distributable Simulations}

One of the most important implications of the STEAMER system is distributability. STEAMER is being designed into a highly portable, low-cost, general purpose piece of hardware. The result is that once the software is accomplished, the system can be spread to a wide variety of sites. Our present cost estimates suggest that the table-top computer to run the steamer system will cost under $\$ 10,000$ in 1983 , when the system is ready for testing.

Because the hardware is general purpose, any other 
STEAMER-like training systems (say, for gas turbines, tactics, nuclear propulsion systems) can be run on the same hardware. Even better, machines nearly capable of running STEAMER are being purchased by some ships already as word processing devices.

The low cost per unit means that STEAMER can be used in conjunction with more traditional high-fidelity simulations, such as those currently used. STEAMER can be used as a test and evaluation device for insuring that students are fully prepared to make maximum use of the high-fidelity system. In addition, one can imagine STEAMER devices stationed at sites in the high-fidelity simulation to provide on-line instruction, as well as to permit students to look "inside" the system to directly observe the consequences of their actions.

\section{CONCLUSION}

The STEAMER project is just finishing its 1 st year in a 5-year program. We have substantial pieces of the math model running on a large machine (Roberts \& Forbus, Note 2) with a suitably flexible graphics interface. We have begun conducting experiments with students learning principles of steam plant operation. We are embarking on an effort to put portions of the
STEAMER system onto a small machine for placement in classroom demonstration situations.

The goal of the STEAMER project is to produce the prototype for a low-cost, portable, inspectable simulation with an embedded computer coach or tutor. We believe these types of training devices have a much wider application than propulsion engineering and will play a major role in meeting the future training needs of the Navy.

\section{REFERENCE NOTES}

1. Forbus, K., \& Stevens, A. Using qualitative simulation to generate explanations (Tech. Note). San Diego, Calif: Navy Personnel Research and Development Center, 1980.

2. Roberts, B., \& Forbus, K. The STEAMER mathematical simulation (Tech. Note). San Diego, Calif: Navy Personnel Research and Development Center, August 1980.

\section{REFERENCES}

DEKLEER, J. Causal and teleological reasoning in circuit recognition. Unpublished doctoral thesis, Massachusetts Institute of Technology, 1979. (a)

DeKleer, J. The origin and resolution of ambiguities in causal arguments. In B. Buchanan (Ed.), Proceedings of the 6th International Joint Conference on Artificial Intelligence. Stanford, Calif: Computer Science Department, Stanford University, 1979. (b) 